\title{
On the Bernoulli free boundary problem and related shape optimization problems
}

\author{
M. HAYOUNI ${ }^{\dagger}$ \\ Institut Elie Cartan, UHP Nancy 1, BP 239, 54506, Vandoeuvre-lès-Nancy, France \\ A. HENROT ${ }^{*}$ \\ Ecole des Mines et Institut Elie Cartan Nancy, BP 239, 54506, Vandoeuvre-lès-Nancy, France \\ AND \\ N. SAMOUH ${ }^{\S}$ \\ Dept. Maths. Fac. des Sciences My Ismail BP 4010 Beni M'Hamed-Meknes and LERMA, EMI, \\ BP 765, Agdal Rabat Maroc, France \\ [Received 14 July 1999 and in revised form 13 July 2000]

\begin{abstract}
This paper deals with the classical Bernoulli free boundary problem. We are interested in solving some shape optimization problems related to this free boundary problem. We prove the continuous dependence of the solution with respect to the data $K$, working with Hausdorff convergence. We can deduce an existence result for a large class of shape optimization problems. Finally, we give some ideas for a numerical method, based on the use of conformal mappings, to solve such problems in two dimensions.
\end{abstract}

Keywords: Bernoulli free boundary, continuous dependence, shape optimization

\section{Introduction}

The Bernoulli free boundary problem is a very classical one in the field of free boundaries. Its popularity comes from the simplicity of its statement together with the fertility of the mathematical questions it involves. Roughly speaking, we can present it as follows. For a given bounded domain $K \subset \mathbb{R}^{N},(N \geqslant 2$, and $K$ is starshaped in this paper), one seeks a larger domain $\Omega$ such that the gradient of the capacitary potential of $\Omega \backslash K$ has a prescribed magnitude on $\partial \Omega$ (the boundary of $\Omega$ ).

The problem arises in various flow laws, and several physical situations, e.g. electrochemical machining and potential flow in fluid mechanics. Standard references, are [1,3,4]; see also [7, 8] and the references therein. We also refer to the paper of Acker and Meyer [2] for a good account of applications in general. A complete overview of theoretical results as well as numerical ones is given in [7].

It is well known that there always exists a solution (at least in a weak sense) to this free boundary problem, see below. Moreover, it is also known that if a classical solution exists and if $K$ (the data) is starshaped, then the solution is unique and starshaped, see $[19,20]$ for the two-dimensional case

\footnotetext{
Email: hayouni@iecn.u-nancy.fr

*Email: henrot@iecn.u-nancy.fr

${ }^{\S}$ Email: samouh@fsmeck.ac.ma
} 
and [2] for the general case. In this paper we are interested in the continuous dependence of the solution of the free boundary problem $\Omega$ when $K$ varies. We work with the Hausdorff topology, which is one of the topologies most often used in the framework of shape optimization. In Section 3, we prove the continuity of the map $K \mapsto \Omega$. We deduce an existence result for a large class of shape optimization problems involving the pair $(K, \Omega)$. Finally, in Section 4 , we give some ideas for a numerical method to solve such problems in two dimensions. It is based on the use of conformal mappings.

\section{Notations and preliminary results}

Let $D_{0}$ be a given bounded and simply connected domain in $\mathbb{R}^{N}(N \geqslant 2)$ and $Q$ a non-negative constant. To any compact set $K \subset D_{0}$ we want to associate an open set $\Omega_{K}$ containing $K$ and a function $u$ (called the capacitary potential of the pair $\left(K, \Omega_{K}\right)$ ) which solve the following classical Bernoulli free boundary problem:

$$
\left\{\begin{aligned}
\Delta u=0 & \text { in } \quad \Omega_{K} \backslash K, \\
u=1 & \text { on } \partial K, \\
u=0 & \text { and }|\nabla u|=Q \quad \text { on } \partial \Omega_{K} .
\end{aligned}\right.
$$

In Remark 2.3, we shall see that for any $K \subset D_{0}$ the open set $\Omega_{K}$ is a subset of some regular bounded and simply connected domain $D$.

There are at least three different approaches to prove existence of a solution for the problem (2.1). The first one, initially due to Beurling [4] (see also [12,14]) uses the original method of subsolutions and supersolutions. A supersolution is an open set $\omega$ containing $K$ such that its capacitary potential has a gradient smaller than $Q$ on $\partial \omega$. A solution of (2.1) is then constructed as a minimal set in the class of supersolutions.

The second method presented by Alt and Caffarelli in [3] consists in studying the variational version of (2.1). For this purpose they introduce the functional $J$ defined on the Sobolev space $H_{0}^{1}(D)$ by

$$
J(v):=\int_{D}|\nabla v|^{2} \mathrm{~d} x+\int_{D} Q^{2} \chi_{v} \mathrm{~d} x
$$

The method consists in minimizing $J$ on the set $V(D, K)$ of functions $v$ in $H_{0}^{1}(D)$ which are equal to 1 quasi-everywhere on $K$ (i.e. the $H^{1}$-capacity of the subset of $K$ on which $v \neq 1$ vanishes: see, for example, [9]). Here and througouht the paper $\chi_{v}$ denotes the characteristic function of the set $\Omega_{v}:=\{v>0\}$.

L. Caffarelli and H. W. Alt prove existence and regularity of an absolute minimum $u$. Then $\Omega_{u}$ is a solution of the free boundary problem (2.1), but the condition ' $|\nabla u|=Q$ on $\partial \Omega_{u}$ ' is to be understood in the following weak sense:

$$
\forall \eta \in W_{0}^{1, \infty}(D), \quad \lim _{\varepsilon \rightarrow 0} \int_{\partial\{u>\varepsilon\}}\left(|\nabla u|^{2}-Q^{2}\right) \nu \cdot \eta \mathrm{d} \sigma=0 ;
$$

where $v$ and $\mathrm{d} \sigma$ denote respectively the unit outward normal to $\partial\{u>\varepsilon\}$ and the $(N-1)$ dimensional area element in $\partial\{u>\varepsilon\}$. They prove also that for $N=2$, the free boundary is regular and therefore ' $|\nabla u|=Q$ on $\partial \Omega_{u}$ ' is satisfied in a classical sense (see below). Note that in [3] $Q$ is a non-negative function. 
A third method has been developed by Acker: it is called the trial free boundary method; see e.g. $[1,2]$. We will not use it here.

Since we are interested in studying dependence properties of the solution of (2.1) with respect to the data $K$, it is convenient to work in a context where we have uniqueness of the solution. The larger class for which we know we have uniqueness is the class of starshaped domains.

THEOREM 2.1 Let $K$ be a compact set in $\mathbb{R}^{N}$ and $\delta>0$ a fixed positive number, we assume that $K$ is starshaped with respect to all points in some ball $B_{\delta}\left(x_{0}\right)$. Then the problem (2.1) has a unique solution $\Omega_{K}$, which is classical, i.e.

$$
\begin{gathered}
\Omega_{K} \text { is of class } C^{\infty}, \quad u \in C^{0}\left(\overline{\Omega_{K} \backslash K}\right), \quad u=1 \text { on } K \quad \text { and } \\
\forall x \in \partial \Omega_{K}, \quad \lim _{\substack{y \rightarrow x \\
y \in \Omega_{K}}}|\nabla u(y)|=Q .
\end{gathered}
$$

Moreover, $\Omega_{K}$ is starshaped with respect to all points in the ball $B\left(x_{0}, \delta\right)$.

Proof. See Theorem 3.2 in [2] (uniqueness comes from the classical Lavrent'ev principle).

REMARK 2.1 Let us remark that in the case $N=2$, we do not need to assume the starshapedness with respect to all the points of the ball. Starshapedness w.r.t a single point is enough to obtain the same result. More precisely, we shall distinguish two cases. In the first one, $K=\{x\}$ which is of zero $H^{1}$-capacity and then $V(D, K)=H_{0}^{1}(D)$; thus $u=0$ is the minimizer of $J$ and then one can consider that $\Omega_{K}=\emptyset$ even if it does not contains $K$. The second case is that of starshaped compact sets such that $K \backslash\{x\} \neq \emptyset$. Thanks to the starshapedness property, for any $y \in K \backslash\{x\}, K$ contains the segment $[x, y]$. Thus, the $H^{1}$-capacity of $K$ could not vanish. Moreover, since $\Omega \backslash K$ satisfies the so-called segment property in two dimensions (see e.g. [6]), the capacitary potential $u$ is continuous up to the boundary.

In the sequel, we use the following notations.

Let $r_{0}>0$ be a fixed number and let us consider the family of compact sets $\mathcal{K}_{N}$ defined as follows:

- for $N=2$,

$$
\mathcal{K}_{2}:=\left\{K \subset D_{0} ; \exists x \in K \text { such that } K \text { is starshaped with respect to } x\right\} ;
$$

- for $N \geqslant 3$,

$$
\mathcal{K}_{N}:=\left\{K \subset D_{0} ; \exists x \in K \text { such that } K \text { is starshaped with respect to } B\left(x, r_{0}\right)\right\} ;
$$

where $B\left(x, r_{0}\right)$ denotes the ball of radius $r_{0}$ centred at $x$.

REMARK 2.2 When $N \geqslant 3$ the compact sets of $\mathcal{K}_{N}$ are uniformly Lipschitz since they satisfy the $\varepsilon$-cone property: see, for instance, [11] or [18].

In this paper, we want to study the map

$$
K \in \mathcal{K}_{N} \longmapsto \Omega_{K},
$$

where $\Omega_{K}$ is the solution of (2.1) for $K$. As a first result, we can prove monotonicity of this map, with respect to set inclusion. 
THEOREM 2.2 Let $\Omega_{K_{1}}$ and $\Omega_{K_{2}}$ denote the solution of (2.1) corresponding to the compact sets $K_{1}$ and $K_{2}$. Then

$$
K_{1} \subset K_{2} \Longrightarrow \Omega_{K_{1}} \subset \Omega_{K_{2}}
$$

REMARK 2.3 Let $\rho_{0}>0$ such that $D_{0} \subset \subset B\left(0, \rho_{0}\right)$. By Theorem 2.2, for any $K \subset D_{0} \subset$ $B\left(0, \rho_{0}\right)$, we have $\Omega_{K} \subset \Omega_{\bar{B}\left(0, \rho_{0}\right)}$. But the solution of $(2.1)$ for $K=\bar{B}\left(0, \rho_{0}\right)$ is a ball $B(0, \rho)$ which can be computed explicitly (see [7]). Therefore, we can take the set $B(0, \rho)$ as a domain $D$ containing strictly all the solutions $\Omega_{K}$, for any $K \subset D_{0}$.

Proof. To prove that $\Omega_{K_{1}} \subset \Omega_{K_{2}}$ it is enough to establish that $\Omega_{K_{2}}$ is a supersolution (in the sense of Beurling) for the problem (2.1) with the data $K_{1}$ (because $\Omega_{K_{1}}$ is the minimal set amongst all the supersolutions).

Let $v_{2}$ and $u_{2}$ the capacitary potential of $\Omega_{K_{2}}$ with respect to $K_{1}$ and $K_{2}$ respectively. Since $K_{1} \subset K_{2}$ we have $\Omega_{K_{2}} \backslash K_{2} \subset \Omega_{K_{2}} \backslash K_{1}$; then, using maximum principle we get $v_{2} \leqslant u_{2}$ in $\Omega_{K_{2}} \backslash K_{2}$. This implies that

$$
\forall x \in \partial \Omega_{K_{2}}, \quad \limsup _{\substack{y \rightarrow x \\ y \in \Omega_{K_{2}}}}\left|\nabla v_{2}(y)\right| \leqslant \limsup _{\substack{y \rightarrow x \\ y \in \Omega_{K_{2}}}}\left|\nabla u_{2}(y)\right|,
$$

because both of $v_{2}$ and $u_{2}$ vanishes on $\partial \Omega_{K_{2}}$. Now since $u_{2}$ is in fact a solution of (2.1), we get

$$
\forall x \in \partial \Omega_{K_{2}}, \quad \limsup _{\substack{y \rightarrow x \\ y \in \Omega_{K_{2}}}}\left|\nabla v_{2}(y)\right| \leqslant Q
$$

what means that $\Omega_{K_{2}}$ is a supersolution. This finishes the proof.

Now, to be able to claim the result of continuity, we need to recall some basic facts about the Hausdorff convergence of sets.

For compact sets, we define the Hausdorff metric by

$$
d\left(K_{1}, K_{2}\right):=\max \left(\max _{x \in K_{1}} \min _{y \in K_{2}}|x-y|, \max _{x \in K_{2}} \min _{y \in K_{1}}|x-y|\right) .
$$

If $\left(K_{n}\right)$ (resp. $\left.\left(\Omega_{n}\right)\right)$ is a sequence of compact (resp. open) subsets of $D$, we say that $\left(K_{n}\right)$ (resp. $\left(\Omega_{n}\right)$ ) converges in the Hausdorff sense to a compact set $K$ (resp. to an open set $\Omega$ ) and we write

$$
K_{n} \underset{n \rightarrow+\infty}{\stackrel{H}{\longrightarrow}} K \quad\left(\text { resp. } \quad \Omega_{n} \underset{n \rightarrow+\infty}{\stackrel{H^{c}}{\longrightarrow}} \Omega\right)
$$

if $\lim _{n \rightarrow \infty} d\left(K_{n}, K\right)=0 \quad$ (resp. $\quad \lim _{n \rightarrow \infty} d\left(\Omega_{n}^{c}, \Omega^{c}\right)=0$ ), where $\Omega^{c}:=\bar{D} \backslash \Omega$.

The main properties of the Hausdorff metric are given in $[11,18]$. Below, we recall some of them we will use in this paper.

LEMMA 2.1 Let $\left(K_{n}\right)$ (resp. $\left.\left(\Omega_{n}\right)\right)$ be a sequence of compact (resp. open) subsets of $D$. Then there exists a compact set $K$ (resp. open set $\Omega$ ) and a subsequence $\left(K_{n_{k}}\right)\left(\operatorname{resp} .\left(\Omega_{n_{k}}\right)\right)$ such that

$$
K_{n_{k}} \underset{k \rightarrow+\infty}{\stackrel{H}{\longrightarrow}} K \quad\left(\text { resp. } \quad \Omega_{n_{k}} \underset{k \rightarrow+\infty}{\stackrel{H^{c}}{\longrightarrow}} \Omega\right) .
$$

Moreover, if $K_{n} \underset{n \rightarrow+\infty}{\stackrel{H}{\longrightarrow}} K$ then 
- $K=\left\{x ; \exists x_{n} \in K_{n}\right.$ such that $\left.\lim _{n \rightarrow+\infty} x_{n}=x\right\}$.

- for all $x$ in $\mathbb{R}^{N}, d\left(x, K_{n}\right) \rightarrow d(x, K)$ when $n \rightarrow \infty$

- for all open set $\omega$ we have: $(K \subset \omega) \Longrightarrow\left(\exists n_{\omega}, n \geqslant n_{\omega} \Longrightarrow K_{n} \subset \omega\right)$.

It is also an easy exercise to prove the following lemma.

LEMMA 2.2 Let $\left(K_{n}\right)$ be a sequence of compact sets in $\mathcal{K}_{N}$. Assume that $K_{n} \stackrel{H}{\rightarrow} K$. Then $K$ belongs to $\mathcal{K}_{N}$.

\section{Continuity}

\subsection{The continuity result}

The main result of this section is the following theorem.

THEOREM 3.1 Let $\left(K_{n}\right)$ be a sequence in $\mathcal{K}_{N}$ which converges, in the Hausdorff sense to a compact $K$. Let us denote by $\Omega_{K_{n}}$ and $\Omega_{K}$ the solutions of the Bernoulli problem (2.1) associated to $K_{n}$ and $K$ respectively. Then

$$
\Omega_{K_{n}} \underset{n \rightarrow+\infty}{\stackrel{H^{c}}{\longrightarrow}} \Omega_{K}
$$

REMARK 3.1 In the particular case of $K=\{x\} \in \mathcal{K}_{2}$, Theorem 3.1 is a consequence of Theorem 2.2. Indeed, if $K_{n} \stackrel{H}{\rightarrow}\{x\}$ with $K_{n} \in \mathcal{K}_{2}$ then $K_{n} \subset \bar{B}\left(x, r_{n}\right)$ where $r_{n}=\max \{|x-y| ; y \in$ $\left.K_{n}\right\} \underset{n \rightarrow 0}{\longrightarrow} 0$. As in Remark 2.3, we have $\Omega_{K_{n}} \subset B\left(x, R_{n}\right)$ where $R_{n}$ is given by $r_{n}=R_{n} \exp \left(\frac{-1}{Q R_{n}}\right)$. Therefore, $R_{n} \underset{n \rightarrow 0}{\longrightarrow} 0$ and $\Omega_{K_{n}} \stackrel{H^{c}}{\longrightarrow} \emptyset=\Omega_{K}$.

Consequently, when $N=2$ we shall prove Theorem 3.1 only in the case of $K \in \mathcal{K}_{2}$ with $K \backslash\{x\} \neq \varnothing$.

Before proving Theorem 3.1 let us recall some useful results.

For any open set $\omega \subset D$ and any $f \in H^{-1}(D) \subset H^{-1}(\omega)$ we denote by $u_{\omega, f} \in H_{0}^{1}(D)$ the unique solution of the Dirichlet problem:

$$
-\Delta u=f \quad \text { in } \quad \omega, \quad u \in H_{0}^{1}(\omega),
$$

that we extend by 0 on $D \backslash \omega$.

We say that a sequence $\left(\omega_{n}\right)$ of open sets $\omega_{n} \subset D \gamma$-converges to $\omega \subset D$ if for any $f \in$ $H^{-1}(D), u_{\omega_{n}, f} \rightarrow u_{\omega, f}$ in $H_{0}^{1}(D)$. It is easy to see that $\omega_{n} \gamma$-converges to $\omega$ if and only if $H_{0}^{1}\left(\omega_{n}\right)$ converges in the Mosco sense to $H_{0}^{1}(\omega)$ in the Hilbert space $H_{0}^{1}(D)$ (see [11,17]), i.e.

(i) $\forall \varphi \in H_{0}^{1}(\omega), \exists \varphi_{n} \in H_{0}^{1}\left(\omega_{n}\right)$ such that $\varphi_{n} \stackrel{H_{0}^{1}(D)}{\longrightarrow} \varphi$;

(ii) $\forall \varphi_{n_{k}} \in H_{0}^{1}\left(\omega_{n_{k}}\right), \quad\left(\varphi_{n_{k}} \longrightarrow \varphi\right.$ in $\left.H_{0}^{1}(D)\right) \Longrightarrow \varphi \in H_{0}^{1}(\omega)$.

We shall use the following sufficient conditions which ensure $\gamma$-convergence.

- If $\left(\omega_{n}\right)$ is a sequence of uniformly Lipschitz domains which converge in the Hausdorff sense to a domain $\omega$, then $\omega_{n} \gamma$-converge to $\omega$, see for instance $[5,11,18]$. Note that this is the case when $\omega_{n}=D \backslash K_{n}, \omega=D \backslash K, K_{n}, K \subset \mathcal{K}_{N}(N \geqslant 3)$ and $K_{n} \stackrel{H}{\rightarrow} K$ according to Remark 2.2. 
- In two dimensions, if the number of connected component of the complementary of $\omega_{n}$ denoted by $\# \omega_{n}^{c}$, is uniformly bounded and if $\left(\omega_{n}\right)$ converge in the Hausdorff sense to a domain $\omega$ then $\omega_{n} \gamma$-converges to $\omega$, see $[11,22]$. Note that this is the case when $\omega_{n}=D \backslash K_{n}, \omega=D \backslash K$, $K_{n}, K \subset \mathcal{K}_{2}$ and $K_{n} \stackrel{H}{\rightarrow} K$. Indeed, $\omega_{n}^{c}=\partial D \cup K_{n}$ and since $D$ is simply connected and $K_{n}$ is starshaped and included in $D_{0} \subset D$ we have $\# \omega_{n}^{c}=2$.

REMARK 3.2 Consequently, for any open subset $\Omega$ of $D$ containing all the compact sets $K_{n}$ and $K$, we have that $\Omega \backslash K_{n} \gamma$-converges to $\Omega \backslash K$. Indeed, extending by 0 the functions in $H_{0}^{1}(\Omega \backslash K)$ we get $H_{0}^{1}(\Omega \backslash K)=H_{0}^{1}(\Omega) \cap H_{0}^{1}(D \backslash K)$ and similarly, $H_{0}^{1}\left(\Omega \backslash K_{n}\right)=H_{0}^{1}(\Omega) \cap H_{0}^{1}\left(D \backslash K_{n}\right)$. Then we conclude using the equivalence between the $\gamma$-convergence and the convergence in the sense of Mosco.

To prove Theorem 3.1 we need the following Lemma:

LEMMA 3.1 Let $\left(K_{n}\right)$ be a sequence of compact sets in $\mathcal{K}_{N}$ and $K \in \mathcal{K}_{N}$ such that $K_{n} \stackrel{H}{\rightarrow} K$. Let $\Omega$ be an open set containing $K$. Let $v_{n}$ (resp. $v$ ) denotes the capacitary potential of $K_{n}$ (resp. $K$ ) relatively to $\Omega$ then $v_{n} \rightarrow v$ in $H_{0}^{1}(\Omega)$.

Proof of Lemma 3.1. For $\delta>0$ small enough, consider the compact set:

$$
K_{\delta}:=\{x \in \Omega ; d(x, K) \leqslant \delta\} .
$$

According to Lemma 2.1, there exists $n_{\delta} \in \mathbb{N}$ such that $K_{n} \subset K_{\delta}$ for $n \geqslant n_{\delta}$. Let $\varphi_{0} \in V\left(\Omega, K_{\delta}\right)$ (i.e. $\varphi_{0} \in H_{0}^{1}(\Omega) ; \varphi_{0}=1$ on $K_{\delta}$ ) so that $\varphi_{0} \in V\left(\Omega, K_{n}\right)$ for $n \geqslant n_{\delta}$. Setting $f:=\Delta \varphi_{0} \in H^{-1}(\Omega)$ we see that for $n \geqslant n_{\delta}$, the functions $u=v_{n}-\varphi_{0}$ and $u=v-\varphi_{0}$ solve respectively the Dirichlet problems

$$
\left\{\begin{array} { l } 
{ - \Delta u _ { n } = f \text { in } \Omega \backslash K _ { n } , } \\
{ u _ { n } \in H _ { 0 } ^ { 1 } ( \Omega \backslash K _ { n } ) }
\end{array} \quad \text { and } \quad \left\{\begin{array}{l}
-\Delta u=f \text { in } \Omega \backslash K, \\
u \in H_{0}^{1}(\Omega \backslash K) .
\end{array}\right.\right.
$$

By the $\gamma$-convergence of $\Omega \backslash K_{n}$ to $\Omega \backslash K$ (see Remark 3.2), we get the strong convergence of the sequence $u_{n}$ to $u$ in $H_{0}^{1}(\Omega)$. Hence, $v_{n} \rightarrow v$ in $H_{0}^{1}(\Omega)$.

Proof of Theorem 3.1. Throughout the proof, we shall denote $\Omega_{K_{n}}$ by $\Omega_{n}$. Let us recall that from the variational approach used in [3], for a fixed $K_{n}$, the solution $\Omega_{K_{n}}$ is obtained as $\Omega_{n}=\left\{u_{n}>0\right\}$ where $u_{n}$ is the minimum on $V\left(D, K_{n}\right)=\left\{v \in H_{0}^{1}(D) ; v=1\right.$ quasi everywhere on $\left.K_{n}\right\}$ of

$$
J(v):=\int_{D}|\nabla v|^{2}+Q^{2} \chi_{v} \mathrm{~d} x
$$

We first prove that $u_{n} \stackrel{H_{0}^{1}(D)}{\longrightarrow} u$ where $u$ is a minimum of $J$ on $V(D, K)$; then we will deduce that $\Omega_{K_{n}} \stackrel{H^{c}}{\longrightarrow} \Omega_{K}$ where $\Omega_{K}=\{u>0\}$.

As in the proof of Lemma 3.1, consider the set $K_{\delta}:=\{x \in D ; d(x, K) \leqslant \delta\}$ and $\varphi_{0} \in V\left(D, K_{\delta}\right)$. Since $K_{n} \stackrel{H}{\rightarrow} K$, we have

$$
\exists n_{\kappa}, \quad \forall n \geqslant n_{\kappa}, \quad K_{n} \subset K_{\delta} \quad \text { and } \quad \varphi_{0} \in V\left(D, K_{n}\right) .
$$


Thus $J\left(u_{n}\right) \leqslant J\left(\varphi_{0}\right)$ which implies, thanks to Poincaré inequality, that $\left(u_{n}\right)$ is uniformly bounded in $H_{0}^{1}(D)$. Therefore there exists $\tilde{u} \in H_{0}^{1}(D)$ and a subsequence still denoted by $\left(u_{n}\right)$ such that

$$
u_{n} \underset{n \rightarrow \infty}{\longrightarrow} \tilde{u} \text { weakly in } H_{0}^{1}(D) \text {, strongly in } L^{2}(D) \text { and a.e. in } D \text {. }
$$

From the 1.s.c. of the norm in $H_{0}^{1}(D)$ for the weak convergence, it comes that

$$
\int_{D}|\nabla \tilde{u}|^{2} \mathrm{~d} x \leqslant \liminf _{n \rightarrow \infty} \int_{D}\left|\nabla u_{n}\right|^{2} \mathrm{~d} x
$$

Since $\chi_{u_{n}}$ is bounded in $L^{\infty}(D)$, there exists $\gamma \in L^{\infty}(D)$ such that $0 \leqslant \gamma \leqslant 1$ and, up to a subsequence, $\chi_{u_{n}} \rightarrow \gamma$ weak- star in $L^{\infty}(D)$. Then,

$$
\int_{D} Q^{2} \gamma \mathrm{d} x=\lim _{n \rightarrow+\infty} \int_{D} Q^{2} \chi_{u_{n}} \mathrm{~d} x
$$

but from the convergence a.e. we get $\chi_{\tilde{u}} \leqslant \gamma$ and therefore,

$$
J(\tilde{u}) \leqslant \liminf _{n \rightarrow \infty} J\left(u_{n}\right) .
$$

Furthermore, since $K_{n} \in \mathcal{K}_{N}$ we get by the same argument as in the proof of Lemma 3.1 that $\tilde{u} \in V(D, K)$. So to get that $\tilde{u}=u$, it remains to prove that, for any $v \in V(D, K), J(\tilde{u}) \leqslant J(v)$.

Let $v \in V(D, K)$. One can assume without loss of generality that $v \geqslant 0$ because $J\left(v^{+}\right) \leqslant J(v)$, where $v^{+}=\max (v, 0)$. Since

$$
\int_{D} \chi_{v} \mathrm{~d} x=\left|\Omega_{v}\right|:=\inf \left\{|O| ; O \text { open set satisfying } \Omega_{v} \subset O \subset D\right\},
$$

there exists a sequence of open sets $O_{\varepsilon}$ such that $\left|O_{\varepsilon}\right| \searrow\left|\Omega_{v}\right|$ as $\varepsilon \rightarrow 0$ and $K \subset \Omega_{v} \subset O_{\varepsilon}$. This implies that $\chi_{O_{\varepsilon}} \rightarrow \chi_{v}$ in $L^{1}(D)$, and therefore

$$
\lim _{\varepsilon \rightarrow 0} \int_{D} Q^{2} \chi_{O_{\varepsilon}} \mathrm{d} x=\int_{D} Q^{2} \chi_{v} \mathrm{~d} x .
$$

By Lemma 2.1, we have $K_{n} \subset O_{\varepsilon}$ for $n \geqslant n_{\varepsilon}$. Thus, one can consider $u_{\varepsilon}^{n}$ the capacitary potential of $O_{\varepsilon}$ relatively to $K_{n}$ defined by

$$
\forall \varphi \in V\left(O_{\varepsilon}, K_{n}\right), \quad \int_{O_{\varepsilon}}\left|\nabla u_{\varepsilon}^{n}\right|^{2} \mathrm{~d} x \leqslant \int_{O_{\varepsilon}}|\nabla \varphi|^{2} \mathrm{~d} x .
$$

Extending $u_{\varepsilon}^{n}$ by 0 outside $O_{\varepsilon}$, we get $u_{\varepsilon}^{n} \in V\left(D, K_{n}\right)$. Thus

$$
J\left(u_{n}\right) \leqslant J\left(u_{\varepsilon}^{n}\right) .
$$

Moreover, for any $n$ we have $\Omega_{u_{\varepsilon}^{n}} \subset O_{\epsilon}$, and from Lemma 3.1, $u_{\varepsilon}^{n} \rightarrow u_{\varepsilon}$ in $H_{0}^{1}(D)$ where $u_{\varepsilon}$ is the capacitary potential of $O_{\varepsilon}$ relatively to $K$. Letting $n \rightarrow \infty$, we get

$$
\limsup _{n \rightarrow \infty} J\left(u_{\varepsilon}^{n}\right) \leqslant \int_{D}\left|\nabla u_{\varepsilon}\right|^{2} \mathrm{~d} x+\int_{D} Q^{2} \chi_{O_{\varepsilon}} \mathrm{d} x .
$$


Then, from (3.1) and (3.3) it comes that

$$
J(\tilde{u}) \leqslant \int_{D}\left|\nabla u_{\varepsilon}\right|^{2} \mathrm{~d} x+\int_{D} Q^{2} \chi_{O_{\varepsilon}} \mathrm{d} x .
$$

But, $v \in V\left(O_{\varepsilon}, K\right)$ because $\Omega_{v} \subset O_{\varepsilon}$. Thus, by the definition of $u_{\varepsilon}$ we have

$$
\int_{D}\left|\nabla u_{\varepsilon}\right|^{2} \mathrm{~d} x \leqslant \int_{D}|\nabla v|^{2} \mathrm{~d} x .
$$

From (3.2) we get $J(\tilde{u}) \leqslant J(v)$ which proves that $\tilde{u}$ minimizes the functional $J$ defined in (2.2) for K.

Now, it remains to prove that $\Omega_{n} \stackrel{H^{c}}{\longrightarrow} \Omega_{u}=\Omega_{K}$. By Lemma 2.1, there exists $\Omega \subset D$ and a subsequence still denoted by $\left(\Omega_{n}\right)$ such that $\Omega_{n} \stackrel{H^{c}}{\rightarrow} \Omega$. Then $\Omega_{n} \backslash K_{n} \stackrel{H^{c}}{\rightarrow} \Omega \backslash K$, and provided that $\Omega \backslash K \neq \emptyset$, we have $\Delta u=0$ in $\Omega \backslash K$. Indeed, for any $\varphi \in C_{0}^{\infty}(\Omega \backslash K)$, i.e. $\operatorname{supp}(\varphi) \subset \Omega \backslash K$. Again by Lemma 2.1, $\operatorname{supp}(\varphi) \subset \Omega_{n} \backslash K_{n}$ for $n$ large enough. But $\Delta u_{n}=0$ in $\Omega_{n} \backslash K_{n}$. Then

$$
\int_{D} \nabla u \nabla \varphi \mathrm{d} x=\lim _{n \rightarrow \infty} \int_{D} \nabla u_{n} \nabla \varphi \mathrm{d} x=0 .
$$

Let us remark that the proof is achieved if we prove that $\Omega_{u} \subset \Omega$. In fact in this case $\Omega_{u} \backslash K \subset \Omega \backslash K$; then $\Delta u=0$ in $\Omega \backslash K, u=1$ on $\partial K$ and $u=0$ on $\partial \Omega$. Hence, by the maximum principle we get $u>0$ in $\Omega$, i.e. $\Omega_{u}=\Omega$.

Let us prove that $\Omega_{u} \subset \Omega$. Let us introduce the positive constant $\delta=d\left(K, \Omega_{u}^{c}\right)>0$. Taking into account that $\Omega_{u}$ is a starshaped domain, it is enough to prove that

$$
E:=\left\{x \in \Omega_{u} ; d\left(x, K_{\frac{\delta}{3}}\right)>\frac{\delta}{3}\right\} \subset \Omega .
$$

In the above formula and in the sequel of this proof, we use the notation $d(x, L)$ to represent the distance of a point $x$ to a compact set $L(d(x, L):=\inf \{|x-y|, y \in L\})$. We assume that this notation is not confusing with the Hausdorff distance already defined.

Note that $K_{n} \subset K_{\frac{\delta}{3}}$ for $n$ sufficiently large, by Hausdorff convergence.

Let $x \in E$; for $n \geqslant n_{x}$, we have $x \in \Omega_{n}$ because $u_{n}(x) \underset{n \rightarrow \infty}{\longrightarrow} u(x)>0$. We have also, $B\left(x, d\left(x, \Omega_{n}^{c}\right)\right) \subset \Omega_{n}$. Now, we have the following alternative.

- Either for a subsequence $n_{k}, d\left(x, \Omega_{n_{k}}^{c}\right)>d\left(x, K_{\frac{\delta}{3}}\right)$. Thanks to the Hausdorff convergence of $\Omega_{n_{k}}$ to $\Omega$, we get $d\left(x, \Omega^{c}\right) \geqslant d\left(x, K_{\frac{\delta}{3}}\right)>0$.

- Or, we have for $n$ large enough, $d\left(x, \Omega_{n}^{c}\right) \leqslant d\left(x, K_{\frac{\delta}{3}}\right)$. Consequently $B\left(x, d\left(x, \Omega_{n}^{c}\right)\right) \subset \Omega_{n} \backslash K_{n}$ and by Corollary 3.6 in [3], there exists a constant $C=C(Q, N)$ such that

$$
u_{n}(x) \leqslant C d\left(x, \Omega_{n}^{c}\right) .
$$

Letting $n \rightarrow \infty$ we get by the Hausdorff convergence of $\Omega_{n}$ to $\Omega$,

$$
0<u(x) \leqslant C d\left(x, \Omega^{c}\right) .
$$

That is $x \in \Omega$ and then $E \subset \Omega$. Since $\Omega$ is the only accumulation point for the sequence $\Omega_{n}$ (for Hausdorff convergence), all the sequence converges to $\Omega$. This finishes the proof. 


\subsection{Application to a shape design problem}

One of the physical contexts which leads to a Bernoulli problem like (2.1) is the optimal insulation. Let $K$ be a given pipeline, then $\Omega_{K}$ represents the optimal insulator, in the sense that it minimizes the heat or current leakage, subject to a given amount of insulating material. It is therefore natural to ask a question such as:

Find the shape of a pipeline $K$ such that the optimal configuration $\left(K, \Omega_{K}\right)$ satisfies some property or minimizes some functional.

In the classical framework of optimal shape design, we are led to consider functionals depending on $K$ through the solution $\left(u, \Omega_{K}\right)$ of the free boundary problem (2.1). Namely, we consider a functional defined as

$$
E(K):=\int_{\Omega_{K} \backslash K} f(x, u, \nabla u) \mathrm{d} x=\int_{D} f(x, u, \nabla u) \chi_{[0<u<1]} \mathrm{d} x
$$

where $f$ satisfies the following assumptions:

$$
\left\{\begin{array}{c}
\qquad: D \times \mathbb{R} \times \mathbb{R}^{N} \longrightarrow \mathbb{R} \text { is a Carathéodory function } \\
(x, s, p) \longmapsto f(x, s, p) \\
\text { (i.e. measurable in } x, \text { continuous in } s \text { and } p) . \\
\text { - There exists } a_{0} \in L^{1}(D), b_{0} \in \mathbb{R}^{+}, c_{0} \in \mathbb{R}^{+} \text {such that } \\
|f(x, s, p)| \leqslant a_{0}(x)+b_{0} s^{2}+c_{0}|p|^{2} .
\end{array}\right.
$$

In this section, we restrict ourselves to the class

$$
\mathcal{K}_{N}:=\left\{K \subset D ; \exists x \in K / K \text { is starshaped with respect to } B\left(x, r_{0}\right)\right\} ;
$$

even for $N=2$. In this class, we have the following property:

LEMma 3.2 Let $\left(K_{n}\right)$ be a sequence of compact sets in $\mathcal{K}_{N}$. Assume that $K_{n} \stackrel{H}{\rightarrow} K$. Then the characteristic functions $\chi_{K_{n}}$ converge to $\chi_{K}$ in $L^{1}(D)$. Moreover, if $\Omega_{K_{n}}$ and $\Omega_{K}$ denote the solutions of (2.1) associated to $K_{n}$ and $K$, the characteristic functions $\chi_{\Omega_{K_{n}}}$ converge to $\chi_{\Omega_{K}}$ in $L^{1}(D)$.

The proof of the first part of this lemma can be found in [5] or [11]. It relies on the uniform Lipschitz regularity of the domains $K_{n}$ and $K$. For the second part, we use Theorem 3.1 and once again the first part for the compact sets $D \backslash \Omega_{K_{n}}$ and $D \backslash \Omega_{K}$.

Now, we claim the existence result.

THEOREM 3.2 Assume that $f$ satisfies hypothesis (3.4) and that

$$
\inf \left\{E(K) ; K \in \mathcal{K}_{N}\right\}>-\infty .
$$

Then the shape optimization problem

$$
\text { Find } K^{*} \text { such that } E\left(K^{*}\right)=\min \left\{E(K) ; K \in \mathcal{K}_{N}\right\}
$$

has a solution. 
REMARK 3.3 In many problems, the functional $E$ is non-negative (e.g. when it comes from a least square formulation). So, in such a case, the assumption $\inf \left\{E(K) ; K \in \mathcal{K}_{N}\right\}>-\infty$ is trivial. In other cases, we can use the fact that the solution $u$ takes its values in $[0,1]$, but it remains to estimate values taken by the gradient. We refer to [14] for such estimations in the convex case, where we prove that the maximum of the gradient inside the domain is achieved on the interior boundary $\partial K$.

REMARK 3.4 Nothing can be said about uniqueness of minimizers for the functional $E$ (consider the case of trivial energies $E=0$ ). In general, uniqueness results are very difficult to prove in shape optimization, see [11].

Proof of Theorem 3.2. We use the standard method of the calculus of variations. Let $K_{n}$ be a minimizing sequence; by Lemma 2.1 and 2.2 we can extract a subsequence (still denoted by $K_{n}$ ) which converges to a compact set $K \in \mathcal{K}_{N}$. According to Theorem 3.1 the corresponding solutions $\Omega_{K_{n}}$ converge for the Hausdorff metric to $\Omega_{K}$ and the capacitary potentials $u_{n}$ converge to $u$ strongly in $H_{0}^{1}(D)$. Now, we can write $E\left(K_{n}\right)$ as

$$
\begin{aligned}
E\left(K_{n}\right) & =\int_{\Omega_{K_{n} \backslash K_{n}}} f\left(x, u_{n}, \nabla u_{n}\right) \mathrm{d} x \\
& =\int_{D} f\left(x, u_{n}, \nabla u_{n}\right) \mathrm{d} x-\int_{K_{n}} f(x, 1,0) \mathrm{d} x-\int_{D \backslash \Omega_{K_{n}}} f(x, 0,0) \mathrm{d} x .
\end{aligned}
$$

Thanks to assumptions (3.4), the map $u \longmapsto \int_{D} f(x, u, \nabla u) \mathrm{d} x$ is continuous on $H_{0}^{1}(D)$ (see e.g. [15]). Therefore

$$
\int_{D} f\left(x, u_{n}, \nabla u_{n}\right) \mathrm{d} x \underset{n \rightarrow \infty}{\longrightarrow} \int_{D} f(x, u, \nabla u) \mathrm{d} x .
$$

Moreover, according to Lemma 3.2, we have

$$
\int_{K_{n}} f(x, 1,0) \mathrm{d} x=\int_{D} \chi_{K_{n}} f(x, 1,0) \mathrm{d} x \underset{n \rightarrow \infty}{\longrightarrow} \int_{D} \chi_{K} f(x, 1,0) \mathrm{d} x=\int_{K} f(x, 1,0) \mathrm{d} x
$$

and similarly

$$
\int_{D \backslash \Omega_{K_{n}}} f(x, 1,0) \mathrm{d} x \underset{n \rightarrow \infty}{\longrightarrow} \int_{D \backslash \Omega_{K}} f(x, 1,0) \mathrm{d} x .
$$

This means that $E\left(K_{n}\right) \underset{n \rightarrow \infty}{\longrightarrow} E(K)$ and the theorem is proved.

\section{Numerical analysis of a shape design problem}

\subsection{Introduction}

We want to present a numerical method to solve a shape design problem like (3.5) where $E$ is any functional of the kind described above.

One of the main tools in such a context is to use shape derivatives (see for instance [21]) together with some gradient or Newton methods to be able to deform an initial domain into a better one. In 
our case, this method seems to be unrealistic. First of all, the dependence of $\Omega_{K}$ on $K$ is quite implicit. Therefore, if we perturb a domain $K$ to $K_{t}$ by a transformation $T_{t}=I d+t V(x)$, it is not clear that the solution $\Omega_{K}$ will also be changed by a transformation $\tilde{T}_{t}=I d+t \tilde{V}(x)$. So, the expression of the derivative of the functional on the free boundary seems not to be available. Moreover, from a computational point of view, such an approach would be very expensive since we would have to compute at each iteration of the algorithm, $\Omega_{K_{n}}$ from $K_{n}$. That means solving as many Bernoulli free boundary problems as iterations.

We are going to present a method, specific to the two-dimensional case, which avoids the drawbacks just mentioned. Roughly speaking, the idea is to use conformal maps to look for both $K$ and $\Omega_{K}$. More precisely, the unknown becoming the conformal map, we will be able to perform any minimization algorithm on these conformal maps to reach the optimal configuration $\left(K^{*}, \Omega_{K^{*}}\right)$. We describe the method in the following section.

\subsection{The numerical approach}

The starting point is to observe that if $K \in \mathcal{K}$ and $\Omega=\Omega_{K}$, then $\Omega \backslash K$ is a doubly connected domain which is conformally equivalent to an annulus

$$
C_{R}:=\{z \in \mathbb{C} ; R<|z|<1\} ;
$$

(where $R$ is a priori unknown). We denote by $\Phi$ the conformal mapping such that $\Phi\left(C_{R}\right)=\Omega \backslash K$, see [16].

Now, if $u$ solves (2.1), then using the isomorphism $x=\left(x_{1}, x_{2}\right) \in \mathbb{R}^{2} \mapsto z=x_{1}+\mathrm{i} x_{2} \in \mathbb{C}$ and writing $u(z)=u\left(x_{1}, x_{2}\right)$, we get that $\hat{u}=u \circ \Phi$ satisfies:

$$
\left\{\begin{aligned}
\Delta \hat{u}=0 & \text { in } C_{R}, \\
\hat{u}=1 & \text { on } \gamma_{R}:=\{z \in \mathbb{C} ;|z|=R\} \\
\hat{u}=0 & \text { and }|\nabla \hat{u}|=Q\left|\Phi^{\prime}\right| \text { on } \gamma_{1}:=\{z \in \mathbb{C} ;|z|=1\}
\end{aligned}\right.
$$

provided that $\Phi$ maps $\gamma_{R}$ on $\partial K$ and $\gamma_{1}$ on $\partial \Omega$. But by an explicit computation, we have that for $z=r \mathrm{e}^{\mathrm{i} \theta} \in C_{R}, \hat{u}\left(r \mathrm{e}^{\mathrm{i} \theta}\right)=\frac{\ln r}{\ln R}$ (since it is the unique solution of the Dirichlet problem $\Delta \hat{u}=0$ in $C_{R}, \hat{u}=1$ on $\gamma_{R}$ and $\hat{u}=0$ on $\gamma_{1}$ ). Then we remark that $\Phi$ and $R$ must satisfy

$$
\left|\Phi^{\prime}\left(\mathrm{e}^{\mathrm{i} \theta}\right)\right|=\frac{1}{Q|\ln R|}=\frac{-1}{Q \ln R} .
$$

Let us now introduce the function defined on $C_{R}$ as

$$
v\left(x_{1}, x_{2}\right):=\ln \left|\Phi^{\prime}\left(x_{1}+\mathrm{i} x_{2}\right)\right| .
$$

It is easy to see that $v$ solves the Dirichlet problem

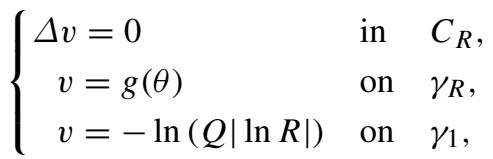

where $g(\theta)=\ln \left|\Phi^{\prime}\left(R \mathrm{e}^{\mathrm{i} \theta}\right)\right|$ for $\theta \in[0,2 \pi]$.

Observe that if $g \in C_{\sharp}^{0}([0,2 \pi])$ (the set of continuous functions such that $g(2 \pi)=g(0)$ ) and $R$ 
(we can prove that for suitable functions $g, R$ is uniquely determined) are given then (4.3) admits a unique solution $v$. If, moreover, one can deduce a unique holomorphic function $\Phi$ satisfying (4.2) and such that $\Phi\left(C_{R}\right)$ is an annulus, taking the form $\Omega \backslash K$ with $\Phi\left(\gamma_{1}\right)=\partial \Omega$ and $\Phi\left(\gamma_{R}\right)=\partial K$, then the functional to minimize can be rewritten as follows:

$$
\begin{aligned}
\mathcal{E}(g, R) & =E(K)=\int_{\Omega \backslash K} f(x, u, \nabla u) \mathrm{d} x \\
& =\int_{C_{R}} f\left(\Phi(z), \hat{u}(z), \frac{\nabla \hat{u}(z)}{\Phi^{\prime}(z)}\right)\left|\Phi^{\prime}(z)\right|^{2} \mathrm{~d} z .
\end{aligned}
$$

So, the problem becomes to minimize a functional $\mathcal{E}$ which depends only on the unknown $(g, R)$, $g \in C_{\sharp}^{0}([0,2 \pi]), R \in \mathbb{R}$. Finally, the conformal map $\Phi$ is obtained from $g$ thanks to a Fourier expansion of $g$ and some straight forward calculations.

One of the main difficulties of this method is to be sure that the holomorphic map $\Phi$ is one-toone. We can prove, using some elementary topologic arguments, that it is the case as soon as $\Phi$ is locally injective (i.e. $\Phi^{\prime} \neq 0$ ) and $\Phi$ is one-to-one on the boundary of the ring (that is to say the image of the two circles are simple curves). See [10] for more details on these questions and some numerical experiments related to this problem.

\section{Acknowledgements}

The work of the third author is partially supported by A.I.845/95.

\section{REFERENCES}

1. ACKER, A. Uniqueness and monotonicity of solutions for the interior Bernoulli free boundary problem in convex $n$-dimensional case. Nonlinear Analysis, TMA 13 (12), (1989) 1409-1425.

2. ACKer, A. \& MEYeR, R. A free boundary problem for the $p$-Laplacian: uniqueness, convexity and successive approximation of solutions. Electronic J. Diff. Eqns 8, (1995) 1-20.

3. Alt, H. W. \& CAfFARElli, L. A. Existence and regularity for a minimum problem with free boundary. J. Reine Angew. Math. 325, (1981) 105-144.

4. Beurling, A. On free boundary problems for the Laplace equation. In Seminars on Analytic Functions I. pp. 248-263. Institute for Advanced Studies Seminars, Princeton (1957).

5. Chenais, D. Sur une famille de variétés à bord lipschitziennes, application à un problème d'identification de domaine. Ann. Inst. Fourier 27(4), (1977) 201-231.

6. Dautray, R. \& Lions, J. L. (eds) Analyse Mathématique et Calcul Numérique, vols I and II. Masson, Paris (1984).

7. Flucher, M. \& RUMPf, M. Bernoulli's free boundary problem, qualitative theory and numerical approximation. J. Reine Angew. Math. 486, (1997) 165-204.

8. Friedman, A. Variational Principles and Free Boundaries. Wiley, (1982).

9. Hedberg, L. I. Spectral synthesis in Sobolev spaces. In: Benedetto, J. J. (ed), Euclidean Harmonic Analysis, Lecture Notes in Mathematics 779. Springer, Berlin (1980).

10. Hayouni, M., Henrot, A., \& Samouh, N. A numerical study of some shape optimization problems, to appear.

11. Henrot, A. \& Pierre, M. Optimisation de forme, to appear.

12. Henrot, A. \& SECK, D. Retour à une ancienne approche pour un problème à frontière libre classique. Publication Mathématiques de Besançon-Analyse non linéaire 15, (1995/97) 29-40.

13. Henrot, A. \& Shahgholian, H. Convexity of free boundaries with Bernoulli type boundary condition. Nonlinear Analysis TMA 28 (5), (1997) 815-823. 
14. Henrot, A. \& Shahgholian, H. Existence of a classical solution to a free boundary problem for the p-Laplace operator I: the exterior convex case. J. Reine Angew. Math. 521, (2000) 85-97.

15. KAVIAN, O. Introduction à la théorie des points critiques et applications aux problèmes elliptiques. Mathématiques et Applications, vol. 13. Springer, Berlin (1993).

16. Lavrent'eV, M. A. \& Chabat, B. Methodes de la Théorie des Fonctions d'une Variable Complexe. Mir, Mosco (1972).

17. Mosco, U. Convergence of convex sets and of solutions of variational inequalities. Adv. Math. 3, (1969) 510-585.

18. Pironneau, O. Optimal Shape Design for Elliptic Systems. Springer, Berlin (1984).

19. TePPER, D. E. Free boundary problem. SIAM J. Math. Anal. 5 (5), (1974) 841-846.

20. TePPER, D. E. On a free boundary problem, the starlike case. SIAM J. Math. Anal. 6 (3), (1975) 503-505.

21. Sokolowski, J. \& Zolesio, J. P. Introduction to Shape Optimization: Shape Sensitivity Analysis. Springer, Berlin Heidelberg (1992).

22. ŠVerÀK, V. On optimal shape design. J. Math. Pures. Appl. 72, (1993) 537-551. 